OAI-PMH: http://www.indteca.com/ojs/index.php/Revista Scientific/oai

Artículo Original / Original Article

\title{
Programa de Educación Ambiental para Contrarrestar los Factores de la Deforestación
}

\author{
Autora: Mairen Danielly Garrido Garrido \\ Universidad Pedagógica Experimental Libertador, UPEL \\ mayren garrido@hotmail.com \\ Barinas, Venezuela
}

\section{Resumen}

El estudio estuvo focalizado con el objetivo de proponer programa de educación ambiental en contrarrestar los factores de la deforestación en las áreas vulnerables de la comunidad del Sector La Vega de los Indios, San Rafael de Canagua, municipio Pedraza, estado Barinas. La investigación enmarcada en el enfoque cuantitativo bajo la modalidad de proyecto factible y en un diseño de campo. La población constituida por ciento cincuenta (150) habitantes y la para efectuar los cálculos de la muestra se utilizó la fórmula propuesta por Palella y Martins (2012), obteniendo un valor de sesenta (60) sujetos. Para la recolección de la información se diseñó una encuesta tipo cuestionario con escalamiento Likert con cinco (05) alternativas de respuestas: Siempre, Casi Siempre, Algunas Veces, Casi Nunca y Nunca. Por lo tanto, el instrumento fue validado por tres (03) expertos en: metodología y educación ambiental. Igualmente, se empleó el coeficiente de consistencia interna de Cronbach (alfa). El análisis de los datos se efectuó por medio de la estadística descriptiva; utilizando la distribución de frecuencia la representación gráfica se empleó el diagrama de barras. El estudio concluye: desconocimiento de los habitantes en función al programa de educación ambiental y los factores de la deforestación. Igualmente, los integrantes de la comunidad tienen la necesidad de aplicar programas de educación ambiental. Se formuló un programa de educación ambiental, el cual se refleja el objetivo específico, contenido, estrategias, actividades, tiempo, recursos, responsables y evaluación. deforestación.

Palabras clave: educación ambiental; medio ambiente; 


\section{Environmental Education Program to Counteract the Factors of Deforestation}

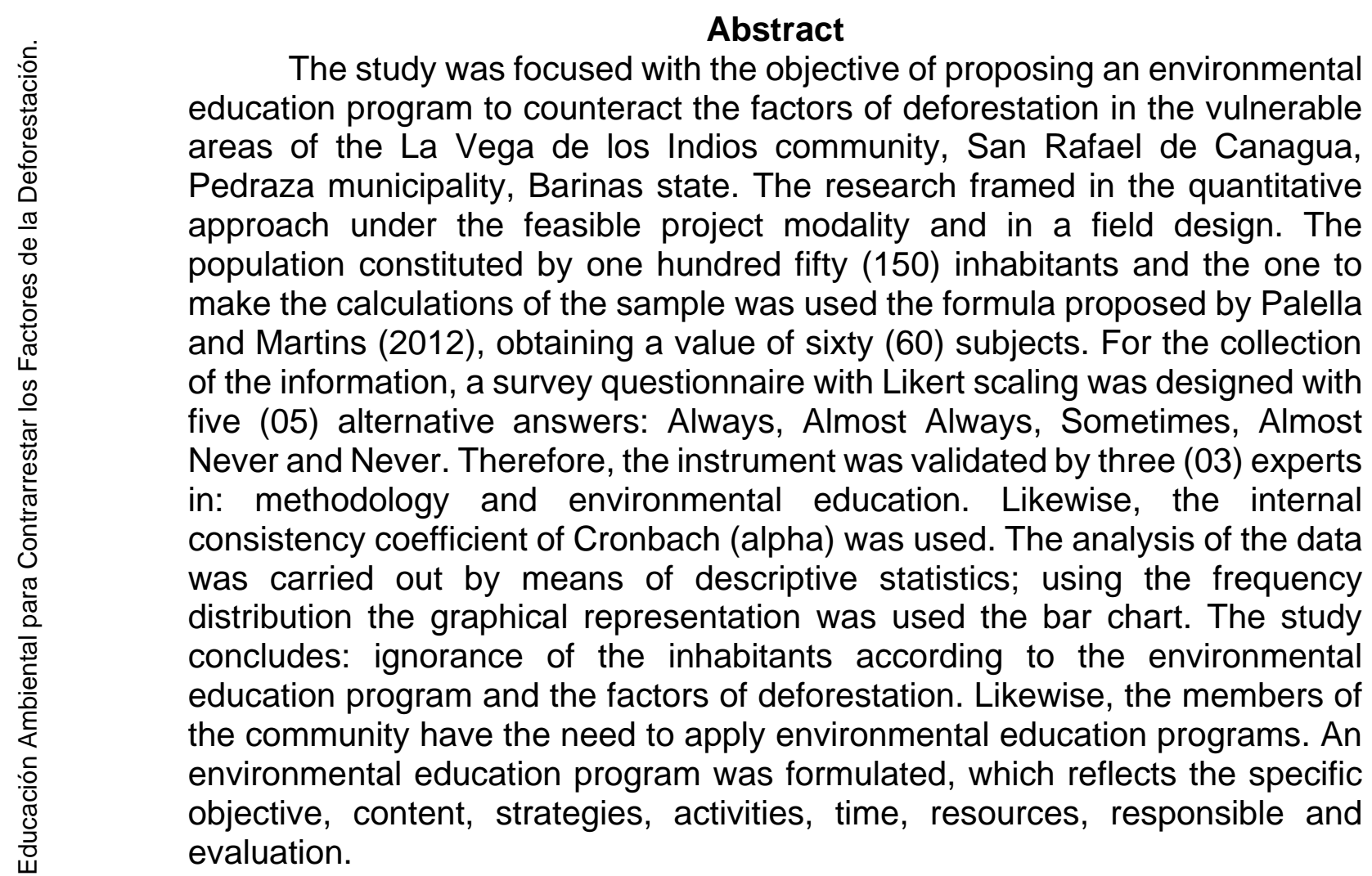

Keywords: environmental education; environment; deforestation. 


\section{Introducción}

La educación ambiental es un campo en continua construcción, su génesis se remonta al viaje inaugural de la hominización transcurriendo por todas las épocas $y$, adquiriendo estatus con la percepción de la crisis ambiental contemporánea. Durante el último siglo, el escenario ambiental entra en los escenarios, políticos, científicos, económicos y educativos, incorporando atribuciones conceptuales; generando espacios para el análisis, interpretación, comprensión y reflexión de acuerdo con los cambios y transformaciones de estos tiempos.

De esta manera, a nivel mundial, emerge la necesidad de una Educación Ambiental. Finalizado los años los setenta, los círculos científicos y académicos y el Consejo Económico y Social de las Naciones Unidas, haciéndose eco de este clima se iniciaron los preparativos que dará pie a la celebración en Estocolmo de una Conferencia Intergubernamental en el año de 1972, en la cual se originó el programa de las Naciones Unidas (PNUMA), dando impulso al Primer Seminario Internacional de la Educación Ambiental.

Por otra parte, el hombre ha ido alcanzados niveles superiores de diferenciación con respecto a otros organismos vivos, fundamentalmente, por la conciencia reflexiva que le acompaña la cual lo ha conducido a instalarse con propiedad sobre los ecosistemas a través de todo un andamiaje adaptativo y, aún más allá, de transformación del medio natural del que se vale. En este interactuar la ciencia ha dado especial importancia a todo el quehacer humano, matizándolo con atributos como un ser eminentemente cultural.

Aún, en la actualidad, luego de reformas insustentables, sigue expresándose la fragmentación en la educación ambiental, reflejo del pensamiento de la ciencia clásica edificada en la modernidad, la cual ha mostrado su incontenible proceso entrópico, ocasionando efectos de grandes magnitudes como lo son: la depreciación de los sentimientos, materiales y espirituales en el conjunto humano, principalmente, los que habitan en las 
poblaciones urbanas, el aumento de la violencia, la delincuencia y el detrimento en la calidad de vida, por citar algunos.

De allí que, los programas de educación ambiental son importantes, razón por la cual accede a la capacitación de opiniones, pensamientos, críticas y actitudes proactivas que fundamentan la disciplina hacia la conservación y mejoras del ambiente. Esto se garantiza cuando los individuos ponen en práctica en acciones que garantizan el bienestar y la protección de la naturaleza.

En este sentido, Moranta (2013): esboza que el programa de educación ambiental es "un proceso integral sistemático y permanente de la información, formación y capacitación formal, no informal e informal, basada en el respeto a toda la forma de vida, por el que las personas, individual y colectivamente" (pág. 34). Esto significa que, representa un proceso sistémico, metódico y permanente del conocimiento en formación, preparación de manera formal e informal en función de fortalecer los valores de respeto, solidaridad, amor, cooperación, unión, trabajo en los individuos y toda la comunidad. Igualmente, profundizar en los cambios de actitudes proactiva en pro del ambiente.

En este orden de ideas, la Organización de las Naciones Unidas para la Agricultura y la Alimentación, citado por Velásquez (2015), plantea que: "los procesos de deforestación a nivel mundial establecen unos estrechos vínculos históricos entre el uso de los bosques y el desarrollo económico y social y entre la destrucción de los bosques y el deterioro económico" (pág. 20). En otras palabras, a nivel internacional la deforestación esta estrecha ligada a la historia en la utilización de los bosques en conjunto de la mano con el progreso de la economía, lo social y la humanidad.

En consecuencia, la deforestación representa un problema más grave en los últimos tiempos. Está temática ha sido de interés a nivel del planeta, debido a la relevancia que tienen los bosques, por todos los beneficios que proporcionan los árboles al ecosistema y a los seres vivos. Es por ello, que en 
los países de Latinoamérica afrontan dificultades en función a lo forestal, razón por la cual ocurren daños severos a la constante presión y depresión como consecuencia de la mala práctica agropecuaria que se efectúan en la zona. La deforestación, consiste en la tala de terrenos, de manera constante, que luego son destinados para otros usos, es una de las transformaciones más incidentes que sufre la superficie terrestre. Al respecto, Pacheco (2011), expone que:

Venezuela es uno de los diez países tropicales que según FAO ha poseído mayor tasa de deforestación en las últimas dos décadas. Situación está preocupante ya que más del $50 \%$ de su territorio está cubierto por bosque, de estos más del $90 \%$ se encuentran en la Amazonía venezolana, que representa un $5,6 \%$ del total de la Amazonía. Estos datos hacen que Venezuela sea consideradas como uno de los 17 países con mayor índice de biodiversidad de la Tierra (pág. 1).

Interpretando al autor, Venezuela es uno de los países que más deforestación existe, motivado que la gran mayoría de las extensiones de terreno están cubierta con bosques; implicando la pérdida progresiva e inalterable de uno de los patrimonios más importantes para el país y por supuesto la proyección para futuras generaciones. No obstantes, de no aplicarse acciones educativas a mediano tiempo que garantice la efectividad de políticas y estrategias en función a la protección, manejo y el aprovechamiento de los recursos forestales.

Por lo tanto, el programa de educación ambiental que contrarreste los factores de deforestación incidirá en el fortalecimiento de los individuos en el fortalecimiento de las actitudes y el comportamiento, en la cual, no solamente, se fortalezca la adquisición de conocimiento y las capacidades, sino en el desarrollo integral del ser, que permita educar para la naturaleza, afrontando con actitud positiva los problemas que tienen que ver con la relación serambiente-sociedad. 
Partiendo de lo antes expuesto, Guillen (2016a): reseña en su trabajo de investigación que: "las comunidades del municipio Barinas carecen de la aplicación de programas de educación ambiental que contribuyan con el mejoramiento del ambiente" (pág. 17). La autora refleja la ausencia de programas en las comunidades destinadas a fortalecer la conservación del ambiente; generando problemas ambientales graves en las localidades.

La situación, antes mencionada, no escapan las comunidades del municipio Pedraza, específicamente el Sector La Vega de los Indios, San Rafael de Canagua, en la cual a través de la observación directa se percibe como lo miembros de dicha comunidad deterioran las áreas vulnerables que se encuentran cercanas a la misma con la quema indiscriminada de las plantas.

Por otra parte, en conversaciones con habitantes de la mencionada comunidad exponen la ausencia de programas de educación ambiental que disminuya la deforestación en la localidad; así como también procesos de formación y sensibilización que conduzcan a razonar la importancia y responsabilidad del ambiente. Además, la presencia de focos de contaminación y deterioro en áreas verdes, aceras, calles avenidas, fachadas de casas y en la conducta de cada habitante al no respetar el espacio físico común de los ciudadanos.

De allí que, la falta de un programa de educación ambiental que contrarreste los factores de la deforestación en áreas vulnerables trae como causas: perdida de cobertura boscosa en la comunidad del Sector La Vega de los Indios, degradación ecológica de los suelos para la producción fértil de productos, altos índice de quema de plantas, arbustos o árboles y la erosión de los suelos. De igual manera, las consecuencias que se puede generar alto índice de contaminación ambiental, desconocimiento de los factores de la deforestación, escasez de agua para el consumo doméstico, destrucción de los bosques, aumento de la seguía, daños a la producción agrícola, 
dificultades en la obtención de alimentos, medicina, aumento de los gases incoloros en el ambiente, desaparición de la naturaleza, los animales entre otros.

La situación planteada motivó el abordaje de la presente investigación, estableciendo como objetivo general: proponer programa de educación ambiental para contrarrestar los factores de la deforestación en las áreas vulnerables de la comunidad del Sector La Vega de los Indios, San Rafael de Canagua, municipio Pedraza, estado Barinas. El estudio plantea las siguientes interrogantes de investigación:

¿Cuál es la situación actual en cuanto a la aplicación del programa de educación ambiental para contrarrestar los factores de la deforestación en las áreas vulnerables de la comunidad del Sector La Vega de los Indios, San Rafael de Canagua, ¿municipio Pedraza?, ¿Cómo será la factibilidad técnica, económica y social de la propuesta del programa de educación ambiental para contrarrestar la deforestación en las áreas vulnerables de la comunidad del Sector La Vega de los Indios?, ¿Cómo será el diseño del programa de educación ambiental programa de educación ambiental para contrarrestar los factores de la deforestación en las áreas vulnerables de la comunidad del Sector La Vega de los Indios, San Rafael de Canagua, municipio Pedraza, estado Barinas?.

\section{Antecedentes, Teoría y Conceptos}

\subsection{Antecedentes}

Los antecedentes son el conjunto de investigaciones, trabajos de ascensos y seminarios que tienen relación directa e indirecta con el tópico que se está investigando. Al respecto, Torracchi (2015): en su estudio titulado "Deforestación y Pérdida de Hábitat en Bosques de Montaña en la Cuenca Alta del Río Zamora (Loja-Ecuador)" (pág. 1). Se planteó como objetivo estudiar las causas de la deforestación y fragmentación de bosques de montaña dentro 
de la cuenca del río Zamora, en la composición y estructura de la diversidad de árboles, líquenes y hepáticas presentes en este tipo de vegetación. La metodología utilizada en el paradigma cuantitativo, tipo de investigación descriptiva apoyada en un estudio de campo, la población constituida por la comunidad de Loja.

El autor concluyó, los bosques montanos experimentados en la provincia de Loja en el sur del Ecuador son ampliamente variables en función a la riqueza de las especies, siendo muchas de ellas variedades que se encuentran en el interior de los bosques, quienes disponen entidades complejas que responden al efecto del borde; en consecuencia, son sensibles a la desintegración forestal.

El antecedente aporta elementos teóricos relacionados con deforestación, causas y consecuencias. La misma representa la ausencia de cobertura forestal, que generan otros tipos de usos, entre ellos: siembra, praderas, nuevos lugares de seres vivos, construcción de viviendas, entre otros aspectos fundamentales que dan origen a las causas y consecuencias en las localidades.

De igual manera, Guillen (2016b): en su estudio titulado "Programa de Educación Ambiental para el Aprovechamiento de los Residuos Sólidos" (pág. 1). La investigación se planteó como objetivo los residuos sólidos en los docentes Educación Primaria de la Unidad Educativa "Herminio León Colmenárez", ubicada en la urbanización Dominga Ortiz de Páez, parroquia Ramón Ignacio Méndez, municipio Barinas, estado Barinas. La naturaleza de investigación se enmarcó en el paradigma cuantitativo. El tipo de investigación se ubicó en la no experimental, transeccional descriptivo y el diseño en la investigación de campo. La población estuvo constituida cincuenta y ocho (58) docentes de Educación Primaria de la institución antes referida, y como muestra la totalidad de la población.

Por consiguiente, la investigadora concluyó: se carece de la utilización 
de un programa de educación ambiental en la integración de actividades hacia el aprovechamiento de los residuos sólidos. Asimismo, tienen la necesidad de aplicar un programa de educación ambiental que contribuya con el mejoramiento de la conservación ambiental. Con poca frecuencia consideran que los programas de educación ambiental se rigen por un conjunto de normativas que se aplican el contexto escolar; también piensan que una característica del programa de educación ambiental la rige la participación.

El estudio contribuyó con elementos teóricos relacionado con el programa de educación ambiental, razón por la cual busca sensibilizar y concienciar a los ciudadanos en la disminución de la deforestación en zonas vulnerables. Además, se tomaron aspectos metodológicos en función al paradigma, tipo, diseño de la investigación y la fórmula para calcular la muestra objeto de estudio. Igualmente, las consideraciones para la elaboración del instrumento.

\subsection{Programa de Educación Ambiental}

El programa representa el motor principal de las accione en el ámbito educativo, en él se reflejan variedad de características focalizadas en el aprendizaje: educandos, cuestionarios, horario escolar, estrategias, recursos y métodos. La Enciclopedia Técnica de la Educación (2012), el programa:

Es un proyecto de acción consistente en la pormenorización de un temario de exigencias generales y mínimas propuesto, con carácter general, a todas las escuelas del país o a un determinado sector de ellas y definido sobre la base de la actividad y la integración (pág. 41).

Es decir, su principal eje se puntualiza en temas generales y específicos dirigidos a todas las instituciones educativas del país o a un sector de ellos, con la finalidad de accionar actividades y la participación en la adquisición de actitudes y hábitos en los cambios del conocimiento, necesidades e intereses 
del ambiente.

No obstante, Añorga (2013): expone que un programa "es la prefiguración de las acciones que los profesores y alumnos han de realizar en el tiempo y el espacio con un fin determinado por el curriculum" (pág. 56). Esto refiere, el programa se conceptualiza en lo deseado reflejado en el perfil del estudiante en cuanto a la educación y filosofía y lo que se puede alcanzar con base a la disponibilidad de recursos en el aula de clase; así como ambientes de trabajo adecuados en los mismos.

Por su parte, Estévez (2012): define al programa como "un conjunto de actuaciones con el objetivo específico de impedir la aparición del problema al que se dirige dicho estudio preventivo" (pág. 21). Dicho programa corresponde a un conjunto de acciones congregadas y cimentadas en propósitos específicos de fijar trabajos y actividades a los individuos que se encuentran comprometidos con el proceso conforme a las potencialidades y capacidades. Además, proporciona información detallada de los objetivos que se persigue; partiendo de los resultados obtenidos en el diagnóstico hacia la consolidación de los fines propuestos.

De acuerdo con lo antes expuesto, el programa constituye uno de los elementos claves en el proceso enseñanza-aprendizaje, que pueda darse, satisfactoriamente, la participación e integración de las actividades en un contexto determinado. Ciertamente se presenta como una guía de trabajo, abierto y flexible que forma parte de la función educativa social e integra todas las acciones realizadas en la institución.

Los programas de educación ambiental deben abarcar aspectos que permitan llevar a cabo pequeñas acciones, que puedan contribuir a una concienciación y mejor vida ecológica, con el fin de alcanzar un efecto multiplicador, de tal manera que las pequeñas acciones produzcan grandes resultados, tanto por efecto acumulador como por sinergia. Al alcanzar este objetivo se cumple con uno de los principios básicos de la misma, lo cual es 
comprender globalmente y actuar localmente.

\subsection{Deforestación en Áreas Vulnerables}

La deforestación, está asociada a los estados de cambio en la utilización del suelo; así como también en la extracción de madera, que pueden generar variaciones centrales en la composición y estructura de la misma; irrumpiendo la capacidad de absorber los sedimentos del entono. Estas acciones son parte del proceso de deforestación, razón por la cual son factores determinantes en la destrucción de las plantas y seres vivos; considerándose superficies deterioradas o deforestadas.

No obstante, Aragón (2013): expone que la deforestación: "ocurre mayormente en países en vías de desarrollo donde el nivel de bienestar de los ciudadanos es un factor crucial en la determinación del tamaño de las áreas deforestadas" (pág. 8). Esto significa, la deforestación comprende la acción humana a través de la tala de árboles, la agricultura y otros factores que juegan en la destrucción de la naturaleza.

Por su parte, Molina (2018): reseña que la educación ambiental "es una nueva forma que facilita la formación de los individuos, donde este es capaz de entender lo complejo que resulta el equilibrio del ambiente, en todas sus facetas" (pág. 130). El autor refleja, la educación ambiental de una manera de visionar la formación de los ciudadanos hacia la armonía del ambiente, desde diferentes contextos, por ello la relevancia de programas destinados a la conservación y preservación del mismo.

\subsection{Factores de la Deforestación}

Los factores de la deforestación son variados y es desigual de una región a otra. Es decir, de acuerdo con el clima y ubicación geográfica del terreno pueden originarse componentes que arriesgan el terreno. Según Reina y De Oro (2005): los principales factores son: "agricultura de la tala y quema, 
ganadería extensiva y pastoreo, utilización de la leña y explotación maderera, presión demográfica, incendios forestales y actividad petrolera” (pág. 32). Los autores refieren algunos orígenes que puede causar la deforestación, a continuación, se describe cada una de las planteadas.

Agricultura de la Tala y Quema: Se puede decir que es uno de los principales agentes que ocasiona la deforestación. El mismo es efectuado por los agricultores que se encuentran localizados en las orillas de los bosques. Esto ocurre en la comunidad de la Vega de los Indios porque las personas que habitan allí talan y queman a diario.

Ganadería Extensiva y Pastoreo: Consiste en la actividad que efectúan los ganaderos en grandes extensiones de terreno, en la cual realizan tala, pastoreo para la cría de ganado; destruyendo el suelo producto del pisoteo del ganado. El pastoreo cuando se ejecuta a campo abierto ocasiona estragos a la vegetación; por lo tanto, es una especie de deforestación que afecta directamente al suelo, cuando la cantidad de ganado repasa la capacidad máxima del suelo y destruya la composición y calidad de los bosques. En la comunidad objeto de estudio se utiliza la ganadería extensiva; ocasionando daños graves al suelo.

Utilización de la Leña y Explotación Maderera: Los troncos de los árboles en forma de leña, desde hace tiempo, ha beneficiado a los individuos, encontrando en ella el combustible ideal para satisfacer las necesidades energéticas. Por lo tanto, el uso desmedido de la madera o leña puede conducir a la destrucción de los suelos, los animales, las plantas y el ecosistema en su totalidad.

\section{Metodología}

\subsection{Naturaleza de la Investigación}

Es importante señalar que los procesos se insertaron en el paradigma cuantitativo de investigación, en vista que se permitió procesar la información 
de modo numérico, empleando especialmente el área de la estadística, en donde se demuestran los hechos con la realización de cálculos. Es decir que, el abordaje de los datos cuantitativos fue estadístico, mediante demostraciones que conducen a la generalización del conglomerado a partir de una muestra de éste, a los que se asigna significado numérico.

\subsection{Tipo de Investigación}

De acuerdo con los objetivos previstos para el desarrollo de esta investigación el cual fue proponer programa de educación ambiental para contrarrestar la deforestación en las áreas vulnerables de la comunidad del Sector La Vega de los Indios, San Rafael de Canagua, municipio Pedraza, estado Barinas, el estudio se enmarcó en la modalidad de investigación de Proyecto Factible. La Universidad Pedagógica Experimental Libertador (2016a), plantea que:

El Proyecto Factible consiste en la investigación, elaboración y desarrollo de una propuesta de un modelo operativo viable para solucionar problemas, requerimientos o necesidades de organizaciones o grupos sociales; puede referirse a la formulación de políticas, programas, tecnologías, métodos o procesos. El proyecto debe tener apoyo en una investigación de tipo Documental, de campo o de un diseño que incluya ambas modalidades (pág. 21).

Por consiguiente, la propuesta del programa de educación ambiental para contrarrestar la deforestación en las áreas vulnerables de la comunidad va contribuir hacia una mejor relación entre las personas que hacen vida dentro del mismo, haciendo énfasis en el desarrollo de los valores organizacionales por este motivo se justificó esta modalidad.

\subsection{Diseño de la Investigación}

El diseño de la investigación que empleó, en este estudio, fue de 
campo, dado que los elementos fueron tomados directamente contexto objeto de estudio. Al respecto, Palella y Martins (2012a), aseguran que el estudio de campo es:

El análisis sistemático de problemas en la realidad, con el propósito bien sea de describirlos, interpretarlos, entender su naturaleza y factores constituyentes, explicar sus causas y efectos, o predecir su ocurrencia, haciendo uso de métodos características de cualquiera de los paradigmas o enfoque de investigación conocidos o en desarrollo (pág. 18).

Interpretando al autor, los datos del estudio fueron tomados de los habitantes de la comunidad del Sector La Vega de los Indios, San Rafael de Canagua, municipio Pedraza, estado Barinas, en la cual a través de la aplicación de una encuesta se podrá evidenciar la necesidad de proponer programa de educación ambiental para contrarrestar los factores de la deforestación en la comunidad del Sector la Vega de los Indios.

\subsection{Población}

La población de una investigación, según Palella y Martins (2012b): plantean que "es el conjunto de unidades de las que se desea obtener información y sobre las que se van a generar conclusiones" (pág. 93). Es decir, la población comprende el grupo de cosas o individuos objeto de estudio. En este sentido, la población, correspondiente a este estudio, estará conformada por ciento cincuenta (150) habitantes de la comunidad del Sector La Vega de Ios Indios, San Rafael de Canagua, municipio Pedraza, estado Barinas. Es importante acotar que se está tomando en cuenta los habitantes jóvenes y adultos quienes proporcionaron la información.

\subsection{Muestra}

La muestra se extrae de la población establecida, por lo que representará un substrato de ella. Al respecto, Márquez (2012), la define como: 
"una parte o subconjunto de la población en las que se supone están presentes las características que identificarán la totalidad" (pág. 101). Es decir, se extrae una muestra de la población objeto de estudio. En consecuencia, para efectuar los cálculos de la muestra se utilizó la fórmula propuesta por Palella y Martins (2012c), quienes plantean: "para poblaciones finitas en la que se introducen un error de estimación, calculado sobre la base del tamaño de la población. Es así como, para poblaciones finitas, el cálculo de la muestra se puede realizar aplicando una fórmula" (pág. 119). Por lo tanto, aplicando la formula determinó que la muestra quedo constituida por sesenta (60) habitantes.

\subsection{Técnicas e Instrumentos de Recolección de Datos}

La técnica empleada fue la encuesta, la cual busca la generalización de lo que plantea el conglomerado con respecto al diagnóstico que se llevará en el contexto del estudio, permitiendo al investigador acercarse a la situación objeto de estudio. De ahí que, la encuesta accedió al acercamiento de la opinión de los habitantes de la comunidad del Sector La Vega de los Indios, San Rafael de Canagua, municipio Pedraza, estado Barinas en cuanto al programa de educación ambiental y los factores de deforestación. El instrumento que se empleó fue un cuestionario porque comprende un conjunto de ítems que se diseñan para obtener los datos que se ameritan para el desarrollo de la investigación. Igualmente, se efectuó utilizando el escalamiento tipo Likert con cinco alternativas de respuestas.

\subsection{Técnicas de Procesamiento y Análisis de Datos}

Las técnicas de análisis de datos, según el Manual para la elaboración de trabajos de grado de especialización y maestrías y tesis doctorales de la Universidad Pedagógica Experimental Libertador (2016b): "se describen en detalles los procedimientos de selección de la muestra, recolección de datos y se hace necesario mencionar y fundamentar el método de análisis de datos 
que va a utilizarse" (pág. 66). Una vez recopilada la información concerniente a la presente investigación, se procedió a su tabulación y análisis mediante la utilización de cuadros estadísticos (frecuencia absoluta y relativa), representada en gráficos de barras.

\section{Resultados}

El diagnóstico representa la primera fase del Proyecto Factible que consiste en recoger los datos directamente de la realidad, en este caso, de los habitantes de la comunidad del Sector La Vega de los Indios, San Rafael de Canagua, municipio Pedraza, estado Barinas. El mismo fue obtenido con la aplicación de un cuestionario relacionado con las variables, dimensiones e indicadores; obteniendo los siguientes resultados:

Gráfico 1. Representación de la distribución de la opinión de los encuestados con relación a la variable Programa de Educación Ambiental.

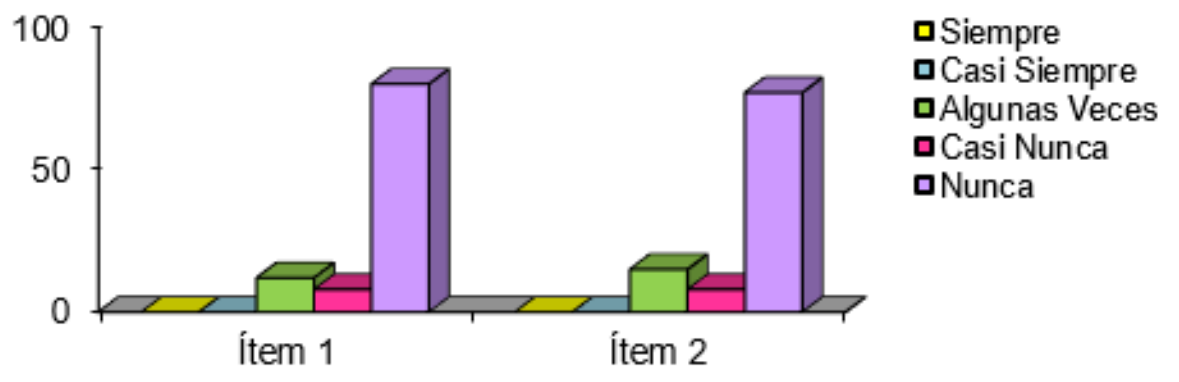

Fuente: La Autora (2017).

En el gráfico 1, en el ítem 1 el 80\% de los encuestados respondieron "Nunca" utilizan un programa de educación ambiental en la integración de actividades para contrarrestar los factores de la deforestación en la comunidad, el 12\% "Algunas Veces" y el $8 \%$ "Casi Nunca". Al respecto, en el ítem 2, el $77 \%$ de los habitantes indicaron "Nunca" aplican programa de educación ambiental para el mejoramiento de la conservación ambiental, el $15 \%$ "Algunas Veces" y el $5 \%$ "Casi Nunca". 
Los resultados inducen a analizar que los habitantes carecen de la utilización de programa de educación ambiental en la integración de actividades para contrarrestar los factores de la deforestación en la comunidad. Así como también, tienen la necesidad de aplicar programa de educación ambiental para el mejoramiento de la conservación ambiental. Esto se debe, posiblemente, a la lejanía de la comunidad y la organización de la comunidad para mejorar la calidad del ambiente.

Gráfico 2. Representación de la distribución de la opinión de los encuestados en relación con la variable Factores de la deforestación en las áreas vulnerables.

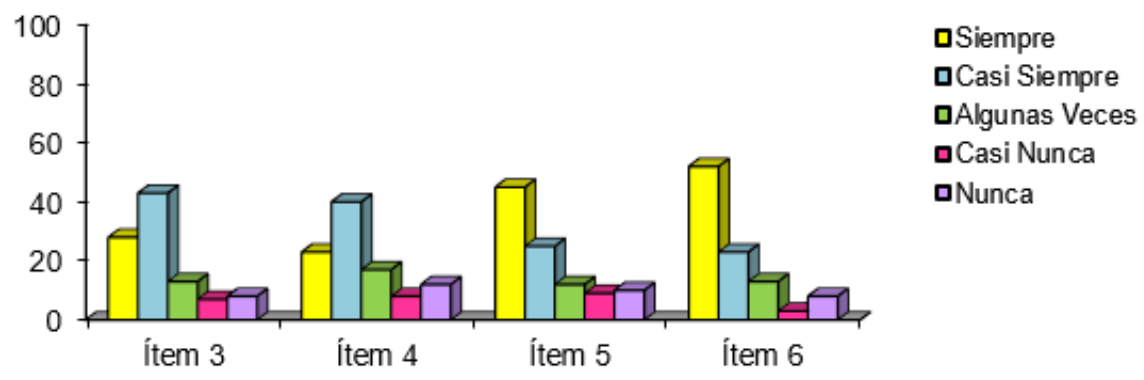

Fuente: La Autora (2017).

En el gráfico 2, en el ítem 3 el 43\% de los habitantes reseñaron "Casi Siempre" considera que en la comunidad se práctica la tala y la quema de los bosques, el 28\% "Siempre", el 13\% "Algunas Veces", el $8 \%$ "Nunca" y el 7\% "Casi Nunca". El ítem 4, el 40\% de los encuestados señalaron "Casi Siempre" en la comunidad se emplea la ganadería de extensión, el 23\% "Siempre", el 17\% "Algunas Veces", el 12\% "Nunca" y el 8\% "Casi Nunca".

De allí que, en el ítem 5 el 45\% de los encuestados indicaron "Siempre" consideran que en la comunidad existe explotación de la madera como factor de la deforestación, el 25\% "Casi Siempre", el 12\% "Algunas Veces", el 10\% "Nunca" y el 8\% "Casi Nunca". El ítem 6, el 52\% respondieron "Siempre" en la comunidad existe presencia de incendios, el 23\% "Casi Siempre", el 13\% 
"Algunas Veces", el 8\% "Nunca" y el 3\% se ubicó en la alternativa "Casi Nunca". Los resultados permiten inferir que los habitantes del Sector La Vega de los Indios, San Rafael de Canagua consideran que existe la práctica de la tala y la quema de los bosques; así como también la ganadería de extensión. Además, consideran que existe la explotación de la manera y un alto índice de incendios forestales. Estos datos conducen a precisar la importancia de la propuesta del programa de educación ambiental para contrarrestar los factores de la deforestación en las áreas vulnerables

\section{Reflexiones Finales}

Los resultados obtenidos en diagnóstico estuvieron centrados en el desconocimiento de los habitantes en función al programa de educación ambiental y los factores de la deforestación. Igualmente, los integrantes de la comunidad tienen la necesidad de aplicar programas de educación ambiental, razón por la cual en dicha comunidad se está la presencia de la quema, la tala de árboles y madera; así como la frecuencia de incendios forestales.

En consecuencia, dichos resultados condujeron al diseño de un programa de educación ambiental para contrarrestar los factores de la deforestación en las áreas vulnerables, como alternativa para el enriquecimiento de la conciencia ambientalista, valores ecológicos y conservación ambiental. De allí que, las actividades planificadas en el programa serán motivantes, estimuladoras; permitiendo la participación y expresión de los integrantes de la institución al proponer acciones puntuales para avanzar hacía el aprovechamiento de residuos sólidos y la conservación del entorno ambiental. Se formuló un programa de educación ambiental, el cual se refleja el objetivo específico, contenido, estrategias, actividades, tiempo, recursos, responsables y evaluación.

De allí la gran importancia del programa de educación ambiental porque permite formar una consciencia social sobre la conservación del ambiente en 
donde intervienen las familias y la comunidad, lo que implica la responsabilidad personal del hombre y su participación colectiva, es decir a ajustado al quehacer comunitario, construyendo mediante la reflexión sobre la acción cotidiana y la transformación permanente.

Igualmente, el programa enfatiza en la formación de los individuos en función de aprender utilizar, concienciadamente, los recursos; satisfaciendo las necesidades e interés actuales y proyectando acciones para el futuro; capacitando a los ciudadanos en valores, ética, actitudes proactivas acorde a los comportamientos y principios de la educación; potenciando las habilidades, destrezas y competencias en aras de mejorar el ambiente. Esto conducirá a un pensamiento conservacionista activo y corresponsable.

\section{Referencias}

Añorga, P. (2013). Programa Educativo. España: Humanitas.

Enciclopedia Técnica de la Educación (2012). Programa. Madrid, España: Océano.

Aragón, L. (2013). Monitoreo de la Deforestación Mediante Técnicas Geomáticas en una Porción de la Región Centro-Norte de México.

Tesis Doctoral. México: Universidad Autónoma de Nuevo León. Recuperado de: http://eprints.uanl.mx/3577/1/1080240823.pdf

Estévez, R. (2012). Programa, Principios y Estructura. Colombia: Trillas. Guillen, A. (2016a,b). Programa de Educación Ambiental para el Aprovechamiento de los Residuos Sólidos. Trabajo de Maestría. Estante 24-7, TM-2017. Barinas, Venezuela: Biblioteca Central. Universidad Nacional Experimental de los Llanos Occidentales Ezequiel Zamora, UNELLEZ.

Márquez, A. (2012). Población y Muestra en la Estadística Aplicada. 2da edición. Colombia: Norma.

Molina, E. (2018). Programa de Reforestación como Alternativa Ambiental 
en la Recuperación de Espacios de Recreación y Esparcimiento. Revista Scientific, 3(7), 121-139. Recuperado de:

https://doi.org/10.29394/Scientific.issn.2542-2987.2018.3.7.6.121-139 Moranta, J. (2013). Programa de Capacitación en Educación Ambiental Dirigido a Docentes. Trabajo Especial de Grado. Maracaibo, Venezuela: Universidad del Zulia. Recuperado de:

http://tesis.luz.edu.ve/tde arquivos/77/TDE-2011-10-07T09:38:05Z1890/Publico/moronta javier.pdf

Pacheco, T. (2011). Deforestación en Venezuela. Caracas, Venezuela: Planeta.

Palella, S., \& Martins, F. (2012a,b,c). Metodología de la Investigación. Segunda Edición. Caracas, Venezuela: Fondo Editorial de la Universidad Pedagógica Experimental Libertador, UPEL-FEDUPEL.

Reina, P., \& De Oro, C. (2005). Vulnerabilidad Ambiental de los Bosques del Municipio de Arauca. Trabajo de Grado, Modalidad de Investigación. Arauca, Colombia: Universidad Nacional de Colombia. Recuperado de: http://www.bdigital.unal.edu.co/633/1/870029.2005.pdf Torracchi, J. (2015). Deforestación y Pérdida de Hábitat en Bosques de Montaña en la Cuenta Alta del Río Zamora. Ecuador, Loja: Departamento de biotecnología-biología vegetal. E.T.S.I. Agronómica, Agroalimentaria y de Biosistemas. Universidad Politécnica de Madrid.

Universidad Pedagógica Experimental Libertador (2016a,b). Manual de trabajos de Grado de Especialización y Maestría y Tesis Doctorales. 5ta. Edición. Caracas, Venezuela: Fondo Editorial de la Universidad Pedagógica Experimental Libertador, UPEL-FEDUPEL. Recuperado de: https://es.slideshare.net/mirnalitaguirrez/manual-upel2016-1pdf

Velásquez, A. (2015). Deforestación a Nivel Mundial. México: Editores. 


\section{Mairen Danielly Garrido Garrido \\ e-mail: mayren garrido@hotmail.com}

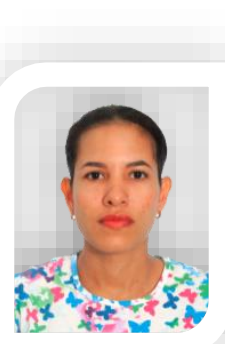

Nacida en Barinas, estado Barinas, Venezuela. Licenciada en Educación, Mención Geografía e Historia, en el año 2010, UNELLEZ, Barinas. Participación en jornadas y talleres: Motivación y comportamiento humano, INCE, Barinas; Evaluación Cualitativa. Instituto de formación "Andrés Bello", Ordenación del territorio y ambiente (UNELLEZ, Barinas), Estrategias para enseñar historia. (UNELLEZ, Barinas). Experiencia laboral como docente de aula en el Liceo Bolivariano "Manuel Palacio Fajardo" dependiente del Misterio para el Poder Popular para la Educación.

El contenido de este manuscrito se difunde bajo una Licencia de Creative Commons ReconocimientoNoComercial-Compartirlgual 4.0 Internacional 